\title{
Clinical improvement associated with targeted interruption of the cerebellothalamic tract following MR-guided focused ultrasound for essential tremor
}

\author{
J. Levi Chazen, MD, ${ }^{1}$ Harini Sarva, MD, ${ }^{2}$ Philip E. Stieg, PhD, MD, ${ }^{3}$ Robert J. Min, MD, MBA, ${ }^{1}$ \\ Douglas J. Ballon, PhD, ${ }^{1}$ Kane O. Pryor, MBBS, ${ }^{4}$ Paul M. Riegelhaupt, MD, PhD, ${ }^{4}$ and \\ Michael G. Kaplitt, MD, $\mathrm{PhD}^{3}$
}

Departments of ${ }^{1}$ Radiology, ${ }^{2}$ Neurology, ${ }^{3}$ Neurosurgery, and ${ }^{4}$ Anesthesia, Weill Cornell Medicine, NewYork-Presbyterian Hospital, New York, New York

OBJECTIVE The objective of this study was to evaluate the utility of diffusion tensor imaging (DTI) tractography-based targeting of the dentatorubrothalamic tract (DRT) for magnetic resonance-guided focused ultrasound (MRgFUS) thalamotomy in patients with essential tremor (ET) and correlate postprocedural tract disruption with clinical outcomes.

METHODS Four patients received preprocedural and immediate postprocedural DTI in addition to traditional anatomical MRI sequences for MRgFUS thalamotomy. Optimal ablation sites were selected based on the patient-specific location of the DRT as demonstrated by DTI (direct targeting) and correlated with traditional atlas-based measurements for thalamic ventral intermediate nucleus (Vim) lesioning (indirect targeting). Fiber tracts were displayed three-dimensionally during the procedure and used in conjunction with clinical signs of tremor control for fine correction of the ablation site. Immediately following the conclusion of the procedure, the MRgFUS head frame was removed and patients were placed in a 32-channel MRI head coil for follow-up DTI and anatomical MRI sequences.

RESULTS All patients had excellent postoperative tremor control and successful pre- and postprocedural DTI fiber tracking of the corticospinal tract, medial lemniscus, and DRT. Immediate postprocedure DTI failed to track the DRT ipsilateral to the lesion site with a preserved contralateral DRT, coincident with substantial resolution of contralateral tremor.

CONCLUSIONS DTI can reliably identify the optimal ablation target and demonstrates tract disruption on immediate postprocedural imaging. A clinical improvement of ET was observed immediately following the procedure, correlating with DRT disruption and suggesting that interruption of the DRT is a consequence of clinically successful MRgFUS thalamotomy. These findings may have utility for both MRgFUS procedure planning in surgically naive patients and retreatment of patients who have previously undergone unsuccessful thalamic Vim lesioning.

https://thejns.org/doi/abs/10.3171/2017.4.JNS162803

KEY WORDS MRgFUS; magnetic resonance-guided focused ultrasound; high-intensity focused ultrasound; diffusion tensor imaging; essential tremor; functional neurosurgery

$\mathrm{M}$ AGNETIC resonance-guided focused ultrasound (MRgFUS) has been successfully applied to the noninvasive treatment of medically refractory essential tremor (ET) and has recently received FDA approval. ${ }^{7,8,10}$ This technique employs a 1024-element phased-array cranial ultrasound unit to generate ablation temperature heating of small intracranial nuclei with millimeter precision. Magnetic resonance imaging guidance provides high-resolution anatomical detail and permits preprocedural and intraprocedural guidance to the optimal ablation site. The thalamic ventral intermediate nucleus (Vim) has proven the ideal target for tremor control using deep brain stimulation (DBS), radiofrequency ablation, Gamma Knife, and MRgFUS techniques. ${ }^{5}$ However, there are significant technical challenges to targeting the Vim, as anatomical delineation of the Vim is limited on

ABBREVIATIONS AC-PC = anterior commissure-posterior commissure; $C R S T=$ combined rating scale for tremor; $C S T=$ corticospinal tract; $D B S=$ deep brain stimulation; $\mathrm{DRT}=$ dentatorubrothalamic tract; DTI = diffusion tensor imaging; $\mathrm{ET}$ = essential tremor; $\mathrm{ML}$ = medial lemniscus; $\mathrm{MMSE}=$ mini-mental state examination; $\mathrm{MRgFUS}=$ magnetic resonance-guided focused ultrasound; QSM = quantitative susceptibility mapping; SWI = susceptibility-weighted imaging; Vim = ventral intermediate nucleus. SUBMITTED December 1, 2016. ACCEPTED April 28, 2017.

INCLUDE WHEN CITING Published online October 20, 2017; DOI: 10.3171/2017.4.JNS162803. 
traditional MRI sequences. As a result, most operators rely on atlas- or coordinate-based techniques for indirect Vim targeting, although these measurements are not patient specific and can vary in individuals based on cerebral atrophy or other morphological alterations. ${ }^{1}$

The potential benefits of diffusion tensor imaging (DTI) have been described in localizing both the corticospinal tract (CST) and medial lemniscus (ML), thereby demarcating the borders of the Vim in an axial plane through the level of the anterior commissure-posterior commissure (AC-PC).$^{20}$ Furthermore, the precise tremor control target has been identified with DTI by mapping the dentatorubrothalamic tract (DRT), which connects the cerebellum with the cerebral cortex through the Vim. ${ }^{4}$ This has been theorized to provide important targeting information and validated with intraoperative recordings in small cohorts. ${ }^{17}$ This has also been applied to noninvasive surgical methods for preprocedure target planning, such as MRgFUS. However, whether DTI-based targeting could be useful as an outcome measure and not only as a targeting method remains unknown. In fact, the value of using this technique for preoperative planning presupposes a functional relevance to ultimate outcome. Because successful lesioning of the Vim should disrupt the DRT, comparison of pre- and postoperative DTI images in the context of clinical outcomes could help confirm the validity of procedural targeting using this method. In this paper we present a novel case series using preoperative DTI targeting of the Vim and immediate postprocedure validation of lesion site and DRT interruption following MRgFUS thalamotomy for ET.

\section{Methods \\ CT Protocol}

Noncontrast CT was acquired on a GE platform using a "BonePlus" filter with interslice resolution of $1 \mathrm{~mm}$ (slice thickness $=1 \mathrm{~mm}$, spacing $=0$ ), matrix of $512 \times 512$, and axial range covering the entire head from the vertex to the skull base. Axial CT images were aligned with the ACPC plane and perpendicular to the midplane. The skull density ratio was calculated from the CT data using a proprietary technique by Insightec.

\section{MRI Protocol}

MRI was obtained at 3-T on a GE 750 platform (software version DV25, revision 2) using a 32-channel head coil. DTI was acquired at 33 directions. Anatomical imaging was performed with 3D T1-weighted BRAVO and 3D T2-weighted CUBE acquisitions that were subsequently reformatted along the AC-PC plane. 2D planar imaging was also performed using axial T2-weighted turbo spin echo and axial susceptibility-weighted imaging (SWI) with quantitative susceptibility mapping (QSM). Imaging parameters are illustrated in Table 1. Imaging was performed prior to the procedure for operative planning and coregistration, immediately following the completion of the MRgFUS thalamotomy, and on postoperative Day 1.

\section{DTI Technique}

Fiber tracking was performed using Brainlab iPlanNet
Cranial (version 3.0). Fractional anisotropy threshold was set to 0.3. Minimum fiber length was $90 \mathrm{~mm}$. Tractography of the CST, ML, and DRT tracts was performed as previously described. ${ }^{20}$

\section{Target Identification}

The neurosurgeon (M.G.K.) and neuroradiologist (J.L.C.) responsible for the MRgFUS ablation arrived at a consensus target on a case-by-case basis at a preoperative planning meeting. Given the novelty of in-vivo use for DTI-based targeting, the authors used their best clinical judgment to select a safe target location considering all available information. As a general principle, a target location in the AC-PC plane was used slightly (approximately $1 \mathrm{~mm}$ ) medial and anterior to the ideal target to account for the uncertainty in MRgFUS spot shape and allow for a safe ablation radius.

\section{MRgFUS Technique}

The study was approved by the Weill Cornell Medicine IRB and informed consent was obtained from all patients. Patients had an intravenous line placed and had blood pressure, peripheral capillary oxygen saturation, electrocardiogram, and exhaled $\mathrm{CO}_{2}$ monitored, and received minimal sedation if required. MRgFUS thalamotomy for treatment of ET has been described previously., ${ }^{2,12}$ Briefly, the patient was placed in a stereotactic frame using 4-point cranial fixation and positioned in a degassed and cooled silicone water bath fitted over the scalp using the Insightec ExAblate Neuro system (software version 6.6). An MR localizer sequence was performed followed by triplanar T2 fast imaging employing steady-state acquisition (FIESTA), angled to the AC-PC plane. Following coregistration of the preprocedure CT and MRI, the ablation site was selected. Adjustments were made by moving the head frame to optimize the geometrical focal point of the MRgFUS unit. Sonications were then performed at graded energies with 2D MR thermometry mapping. After serially adjusting the focal point in reference to the phase-encoding direction in 3 planes using low-energy sonications (approximately $40^{\circ}-45^{\circ} \mathrm{C}$ ), the treatment was initiated by gradually increasing temperature through successive rounds of therapy at the target site. Following each sonication, neurological testing was performed to evaluate tremor response with careful attention paid to abnormal motor or sensory alterations. After a positive neurological response was obtained and 3 or more treatments of the optimal focal point with temperatures approximating $60^{\circ} \mathrm{C}$ (but at least $>55^{\circ} \mathrm{C}$ ) achieved, the treatment was terminated.

\section{Clinical Neurological Evaluation}

Neurological evaluation was performed by a boardcertified neurologist with fellowship training in movement disorders (H.S.). According to Insightec eligibility criteria, patients had severe intentional tremor despite optimal medical therapy or could not tolerate first-line medications (propranolol and primidone) due to side effects. Baseline testing included the combined rating scale for tremor (CRST) score, mini-mental state examination (MMSE), and spiral drawings. The CRST consists of 3 
TABLE 1. MRI protocol

\begin{tabular}{|c|c|c|c|c|c|c|c|c|}
\hline Sequence $^{*}$ & Matrix & $\begin{array}{c}\mathrm{TR} \\
\text { (msec) }\end{array}$ & $\begin{array}{c}\mathrm{TE} \\
\text { (msec) }\end{array}$ & $\begin{array}{c}\mathrm{Tl} \\
\text { (msec) }\end{array}$ & $\begin{array}{l}\text { FOV } \\
(\mathrm{cm})\end{array}$ & $\begin{array}{l}\text { Slice Thickness } \\
(\mathrm{mm})\end{array}$ & Imaging Options† & $\begin{array}{l}\text { Acquisition Time } \\
\text { (min:sec) }\end{array}$ \\
\hline 3D Sagittal T2 CUBE & $256 \times 256$ & 3000 & 70 & None & 25.6 & 1 & $\begin{array}{l}\text { NPW, EDR, Fast, ZIP512, } \\
\text { ZIP2, FR, ARC }\end{array}$ & $4: 45$ \\
\hline 3D Sagittal T1 BRAVO & $256 \times 256$ & 10 & Minimum & 450 & 25.6 & 1 & EDR, Fast, IrP, ZIP2, ARC & $4: 25$ \\
\hline Axial T2 FSE & $384 \times 256$ & 2700 & 110 & None & 24.0 & 2 & FC, EDR, TRF, Fast, FR & $3: 45$ \\
\hline Axial QSM/SWI & $416 \times 320$ & 30 & Minimum full & None & 24.0 & 3 & FC, Fast, Asset & $3: 30$ \\
\hline Axial DTI (33-direction) & $128 \times 128$ & 7500 & Minimum & None & 24.0 & 2.5 & EDR, EPI, DIFF, Asset & $5: 00$ \\
\hline
\end{tabular}

FOV = field of view; FSE = fast spin echo.

* All imaging was performed with zero-slice spacing on a 3-T GE 750 MRI platform.

$\dagger$ Options listed refer to unique imaging options specific to the GE platform.

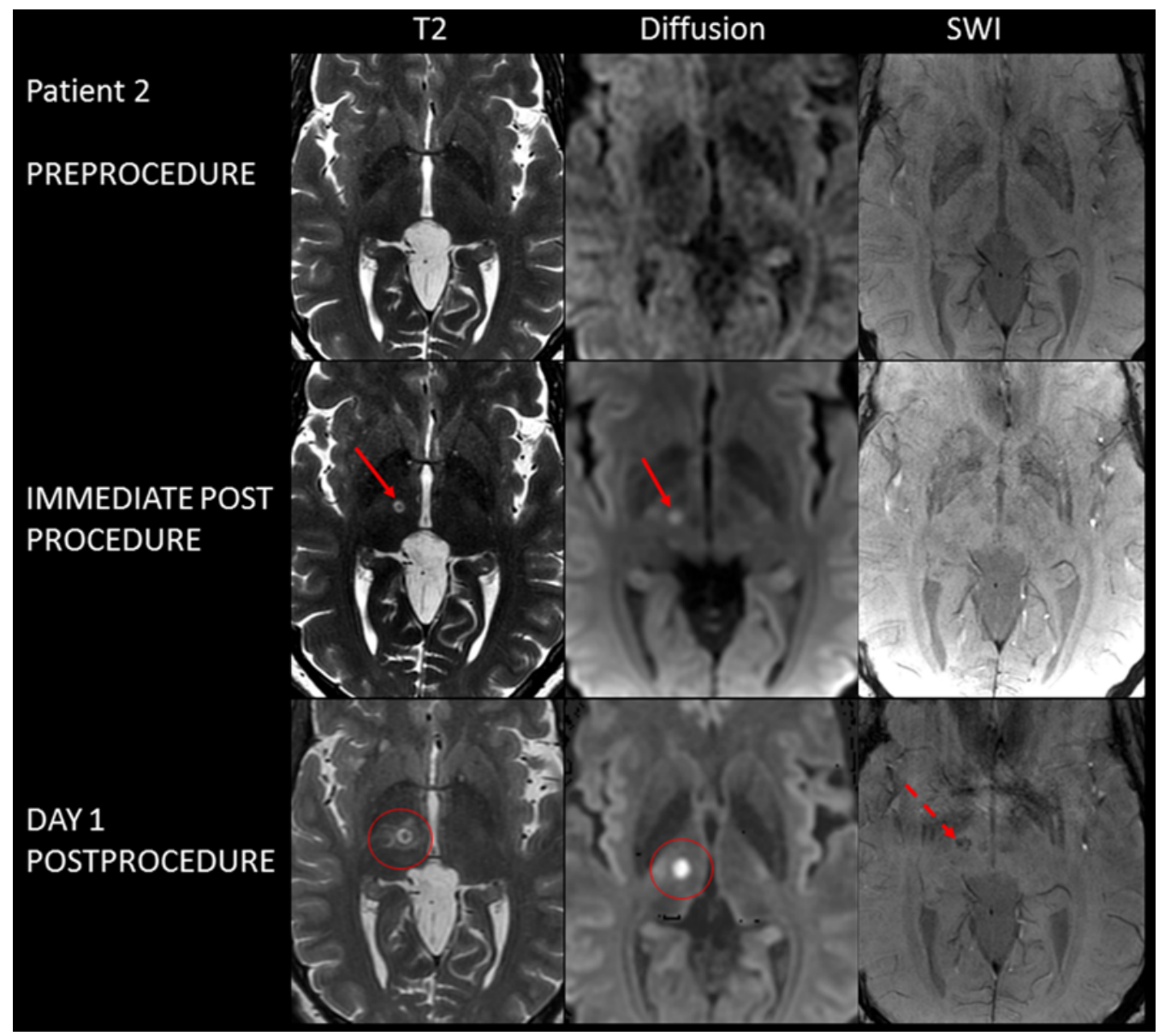

FIG. 1. Pre- and postablation MRI images for Case 2. Solid arrows show the lesion in the right Vim. Imaging performed 24 hours postprocedure reveals increased edema and diffusion signal (circle) in addition to new petechial hemorrhage (dashed arrow).

parts: Part A (tremor localization/severity) establishes the presence of orthostatic tremor and quantifies the tremor at rest, including postural effects and changes with action; Part B (specific motor tasks/function rating) scores the action tremor of the upper extremities while writing and pouring; and Part $\mathrm{C}$ (functional disabilities resulting from the tremor) evaluates for disability from the tremor while speaking, eating, bringing liquids to the mouth, during hygienic care, dressing, and working. ${ }^{11}$ The CRST is a validated, reliable evaluation tool for ET and is recommended for use by the International Parkinson's Disease and Movement Disorders Society. ${ }^{6}$ All included patients were required to have a minimum MMSE score of 25/30. Intraprocedural testing included serial Archimedes spiral drawing evaluations. Archimedes spirals are sensitive and specific for detecting ET and correlate well with tremor severity. ${ }^{13}$ Follow-up clinical visits included a reassessment of CRST, MMSE, and spiral drawings. 


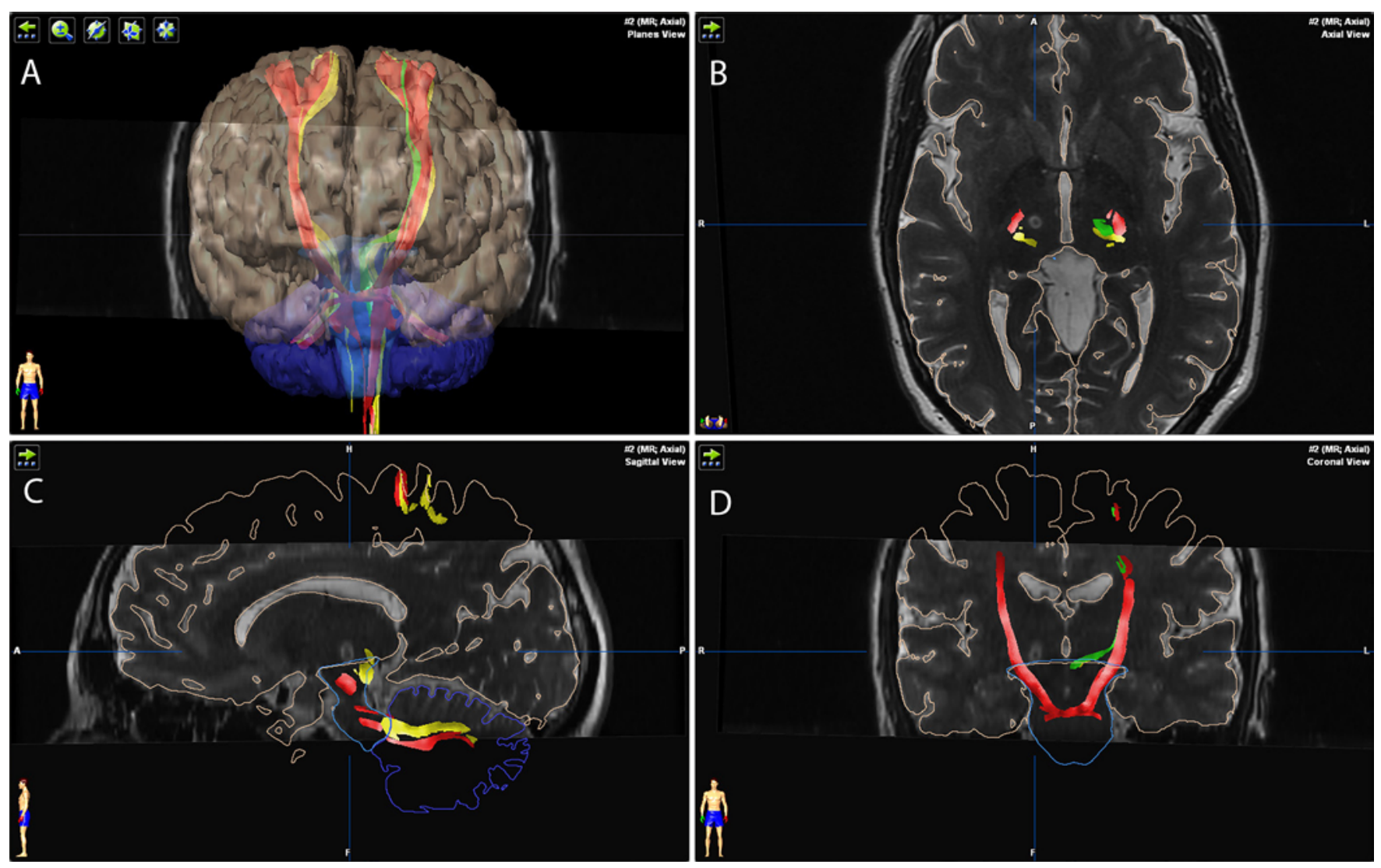

FIG. 2. Case 2. Postablation DTI tractography overlaid on a T2-weighted sequence showing 3D (A), axial (B), sagittal (C), and coronal (D) multiplanar reformations. CST (red), ML (yellow), and corticothalamic (DRT) tract (green) are shown from DTI. Volume-rendered whole brain (tan) and brainstem (blue) outlines are also displayed.

\section{Results}

Preprocedure MRI examinations did not reveal clinically significant abnormalities. Thalamotomy ablation sites were selected using a combination of traditional indirect targeting techniques by an experienced functional neurosurgeon (M.G.K.) with confirmation by DTI maps created by the neuroradiologist (J.L.C.). Adjustments to the target performed during the MRgFUS procedure were guided by the clinical response to low-temperature elevations combined with the location of the DRT relative to the CST and ML. Immediate postprocedure MRI showed a small focus of diffusion signal abnormality corresponding to the thalamic Vim. On the postprocedure Day 1 MRI, the focus of diffusion signal abnormality expanded along with increased surrounding T2-weighted hyperintense edema and susceptibility hypointensity (representing internal microhemorrhage). Representative pre-ablation, immediate postablation, and postoperative Day-1 postabalation MR images are shown in Fig. 1. To determine whether the changes in axial images at the target represent a complete disruption of the DRT without loss of the surrounding tracts, 3D and multiplanar tractography was performed. In each case, this confirmed that MRgFUS-mediated Vim thalamotomy disrupted the entire DRT while the CST and ML were preserved. Rep- resentative 3D and multiplanar tractography information is displayed in Fig. 2.

All patients experienced significant clinical improvement both subjectively and by physician evaluation. Hand tremor severity score was graded on a 15-point scale from Part A of the CRST. Three of 4 patients had a 0/15 CRSTA score on postablation assessment; 1 patient had a 1/15 postablation score. Patient demographic and clinical data are shown in Table 2.

DTI tractography identified the CST, ML, and DRT in all patients bilaterally prior to the procedure. On the immediate postablation DTI tractography, the DRT in the treated hemisphere could no longer be tracked successfully in any of the patients, corresponding with successful tract disruption. The DRT in the contralateral hemisphere, and the CST and ML bilaterally, were successfully tracked following the procedure in all patients and these appeared largely unchanged compared with their pre-lesion DTI. Pre- and postabalation DTI tractography along with corresponding Archimedes spiral evaluations for all patients are shown in Figs. 3-6.

\section{Discussion}

This study reports loss of DRT fiber tracking following MRgFUS thalamotomy in 4 patients associated with 
TABLE 2. Patient characteristics, target measurements, and CRST scores

\begin{tabular}{|c|c|c|c|c|c|c|c|}
\hline \multirow{2}{*}{$\begin{array}{l}\text { Case } \\
\text { No. }\end{array}$} & \multirow{2}{*}{$\begin{array}{l}\text { Age (yrs), } \\
\text { Sex }\end{array}$} & \multirow{2}{*}{$\begin{array}{l}\text { Affected } \\
\text { Hand }\end{array}$} & \multicolumn{2}{|c|}{ Target Measurement (lateral/AP) } & \multicolumn{2}{|c|}{ CRST Hand Tremor Severity Subscore } & \multirow{2}{*}{$\begin{array}{l}\text { Posttreatment MD-evaluated } \\
\text { Immediate \% Improvement }\end{array}$} \\
\hline & & & Via Atlas $(\mathrm{mm})^{*}$ & Via DTI $(\mathrm{mm})^{*}$ & Pretreatment & Posttreatment & \\
\hline 1 & $77, M$ & Rt & $15.5 / 9.0$ & $15.3 / 9.0$ & $4 / 15$ & $1 / 15$ & 85 \\
\hline 2 & $52, \mathrm{~F}$ & $\mathrm{Lt}$ & $13.5 / 8.0$ & $12.4 / 7.2$ & $5 / 15$ & $0 / 15$ & 90 \\
\hline 3 & $71, M$ & $\mathrm{Rt}$ & $14.5 / 7.6$ & $14.4 / 7.4$ & $3 / 15$ & $0 / 15$ & 85 \\
\hline 4 & $57, \mathrm{M}$ & Lt† & $14.0 / 7.5$ & $13.7 / 7.2$ & $3 / 15$ & $0 / 15$ & $90-95$ \\
\hline
\end{tabular}

$\mathrm{AP}=$ anteroposterior; $\mathrm{MD}=$ medical doctor .

* AP measurements relative to the posterior commissure in the AC-PC plane.

$\dagger$ Patient is right-handed but requested treatment of the left hand due to increased tremor severity.
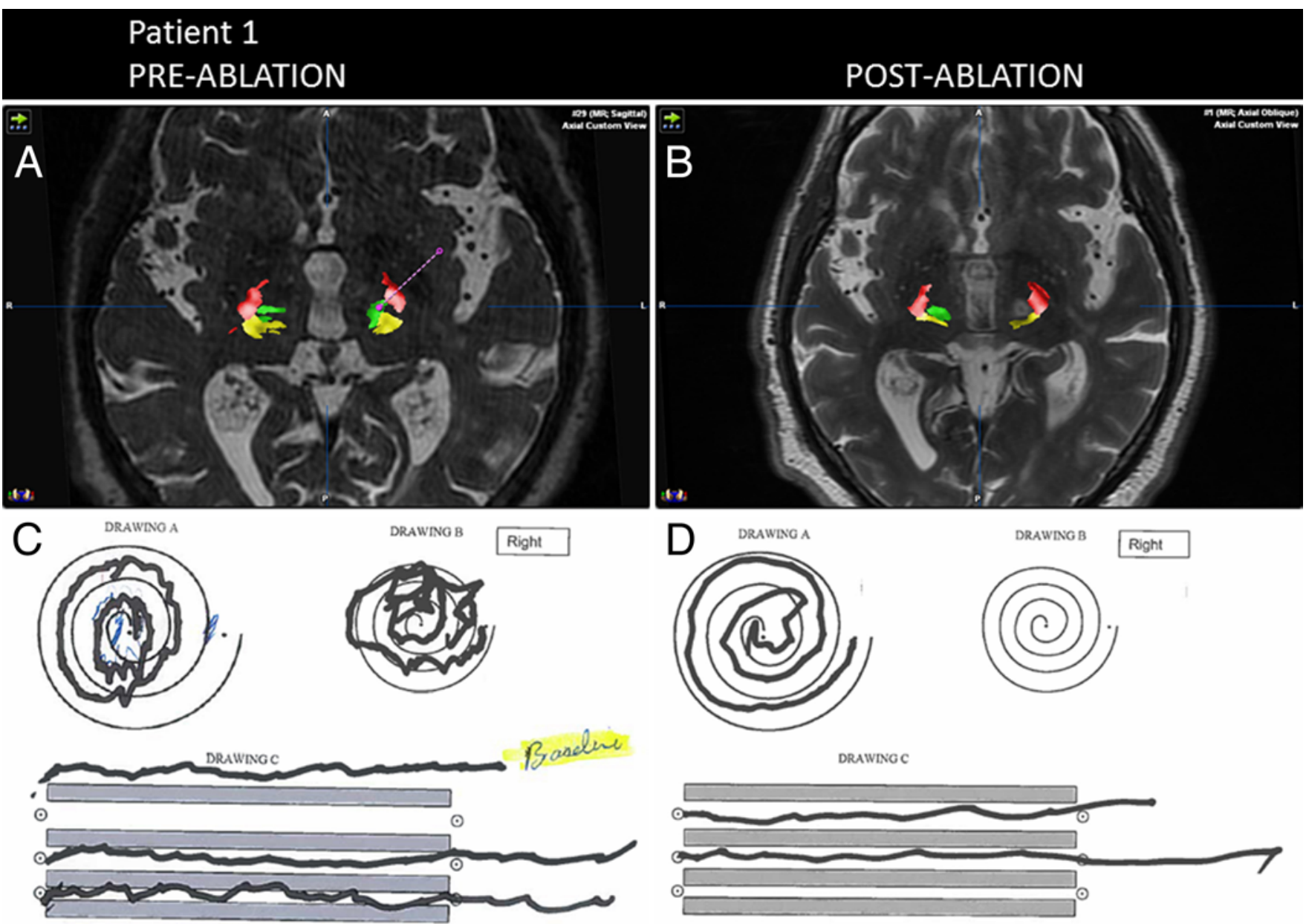

DRAWRoc

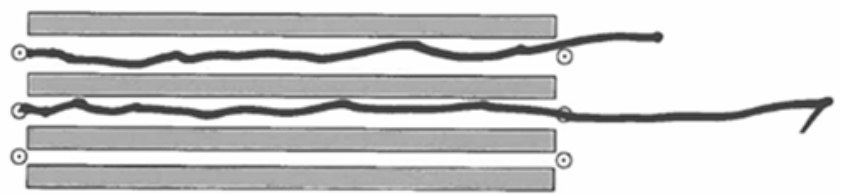

FIG. 3. A and B: Pre- and postablation DTI tractography overlaid on T2-weighted sequences for Case 1. CST (red), ML (yellow), and corticothalamic tract (green) are shown from DTI. Postablation imaging reveals the T2-weighted hyperintense MRgFUS lesion in the location of the right DRT that could no longer be successfully tracked. C and D: Results of the pre- and postprocedure Archimedes spiral drawing tests.

substantial reduction in contralateral intention tremor. All patients underwent successful preprocedural fiber tracking of the CST, ML, and DRT. Furthermore, immediate postprocedure DTI failed to track the DRT ipsilateral to the lesion site while the contralateral DRT remained intact, suggesting immediate fiber track disruption from the procedure. These imaging findings provide a direct cor- relate to the clinical improvement of tremor observed at the termination of the procedure. This supports previous studies that have used this method to augment traditional preprocedure target identification and suggests that disruption of the DRT is a consequence of clinically successful MRgFUS thalamotomy.

The selection of appropriate Vim coordinates has been 


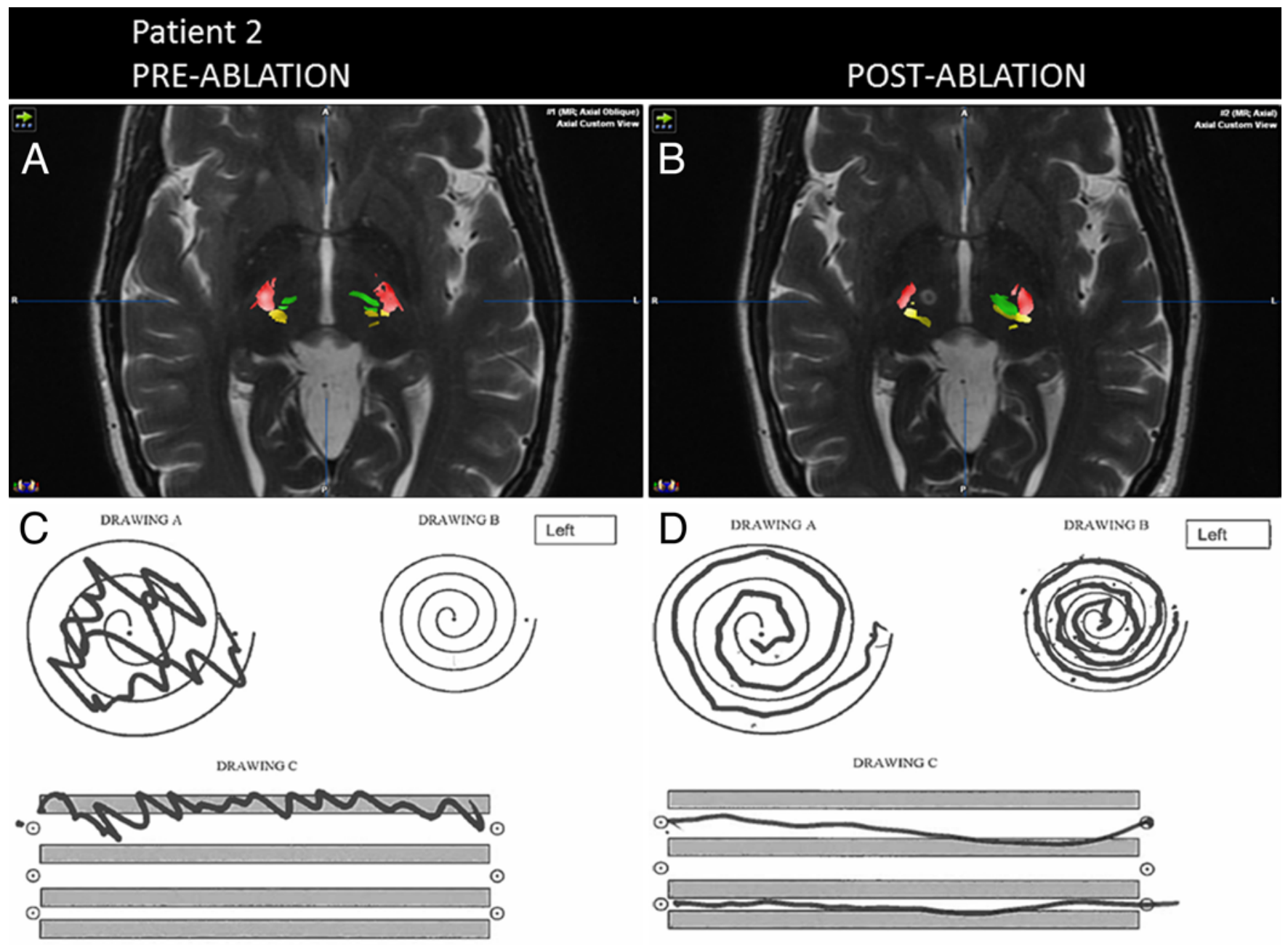

FIG. 4. A and B: Pre- and postablation DTI tractography overlaid on T2-weighted sequences for Case 2. CST (red), ML (yellow), and corticothalamic tract (green) are shown from DTI. Postablation imaging reveals the T2-weighted hyperintense MRgFUS lesion in the location of the right DRT that could no longer be successfully tracked. C and D: Results of the pre- and postprocedure Archimedes spiral drawing tests.

an ongoing challenge in ET treatment, with the largest body of research related to DBS. ${ }^{14}$ Atlas-based targeting (the indirect method) assumes similar anatomy among patients and may vary significantly from image-based coordinate targeting (the direct method).$^{18}$ Given anatomical variations among patients from a variety of conditions, including hydrocephalus and atrophy, a direct patient-specific approach to coordinate selection has inherent benefits. In our cohort, indirect and direct coordinates were calculated independently and showed similar values, further increasing confidence of appropriate site selection. However, when intraprocedural adjustments were necessary due to lack of initial response, knowledge of the DTI tracks to be avoided (CST, ML) and to be targeted (DRT) was subjectively helpful to the authors when deciding upon the subsequent ablation target. We anticipate direct DTI-based coordinate selection will show further benefits in patients who have failed prior ablation techniques or have a recrudescence of tremor.

Independently planned anatomical- and atlas-based co- ordinates demonstrated close agreement with DTI-selected ablation sites. DTI-based coordinate selection showed the epicenter of the DRT slightly posterior and medial to the atlas-based coordinates. However, most operators believe an anterior and medial site to be a safer starting point given the increased buffer between the CST and ML. However, selecting a site that is excessively anteromedial may not produce a clinical tremor response and may prolong the procedure. We believe the DTI information aids the preprocedural and intraprocedural planning by illustrating safe margins and calibrating ablation sites to the patient-specific anatomy. It would be highly desirable to expand these findings and test intraprocedural DTI during the intermediate lesioning steps to help guide subsequent attempts at lesioning. This could be particularly useful when clinical information is vague and does not clearly suggest next steps. However, current MRgFUS technology requires the use of a body coil embedded in the MRI table, rather than a dedicated head coil, which precludes effective DTI acquisition during the procedure. 

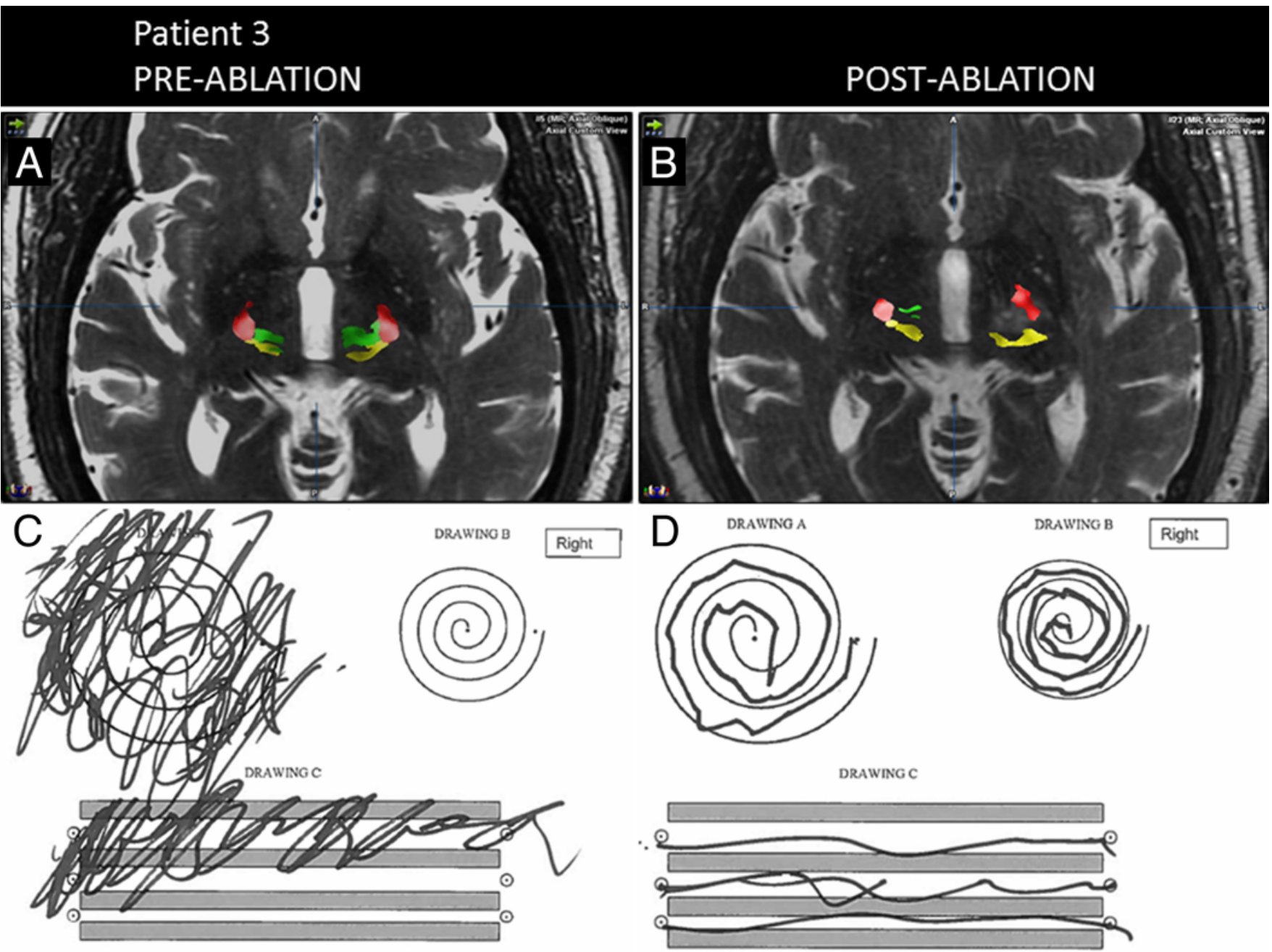

FIG. 5. A and B: Pre- and postablation DTI tractography overlaid on T2-weighted sequences for Case 3. CST (red), ML (yellow), and corticothalamic tract (green) are shown from DTI. Postablation imaging reveals the T2-weighted hyperintense MRgFUS lesion in the location of the left DRT that could no longer be successfully tracked. C and D: Results of the pre- and postprocedure Archimedes spiral drawing tests.

In practice, precise ablation may be challenging due to physical limitations of the cranial vault in individual patients. Although all patients were screened for skull density ratio (minimum 0.4 per Insightec protocol requirements), differences in geometry and total number of elements in the final procedure plan may cause irregularities in the focal spot. ${ }^{3}$ Furthermore, the MR thermometry maps can only display planar information that is reliable in the phase-encoding direction, making evaluation of a 3D ablation site challenging. ${ }^{9,15,16}$ The complex spatial characteristics of the ablation further emphasize the benefit of DTI data, which is displayed in 3 imaging planes and can be manipulated during the procedure. Advances in the MRgFUS imaging technology to allow 3D thermometry maps in real time, combined with active fusion to the DTI imaging, could help overcome the limitation of the body coil and improve the intraprocedural utility of DTI.

When performing the MRgFUS thalamotomy, the ablation spot shape created by the 1024-element array varies considerably based on the laterality and element blocking. Elements are blocked, for example, if the sound energy would travel through air or a fold in the water membrane. As such, the number of elements involved in the ablation varies but was consistently greater than the 800 -element minimum recommended by the manufacturer. This consideration, along with differences in patient skull thickness, results in a complex ablation spot that must be closely monitored throughout the ablation with multiple planes of MR thermometry. As such, the operators found the DTI information helpful to display during the procedure and adjust based on the course of the fiber tracts. For example, the fiber tracking reveals that the CST courses medially below the AC-PC plane as displayed in the coronal plane (Fig. 2). As such, when the coronal MR thermometry map showed an oblong spot shape that extended inferolaterally, the authors used this information to adjust the subsequent ablation spots in a superomedial direction to avoid motor impairment. The authors found this 3D tractography 


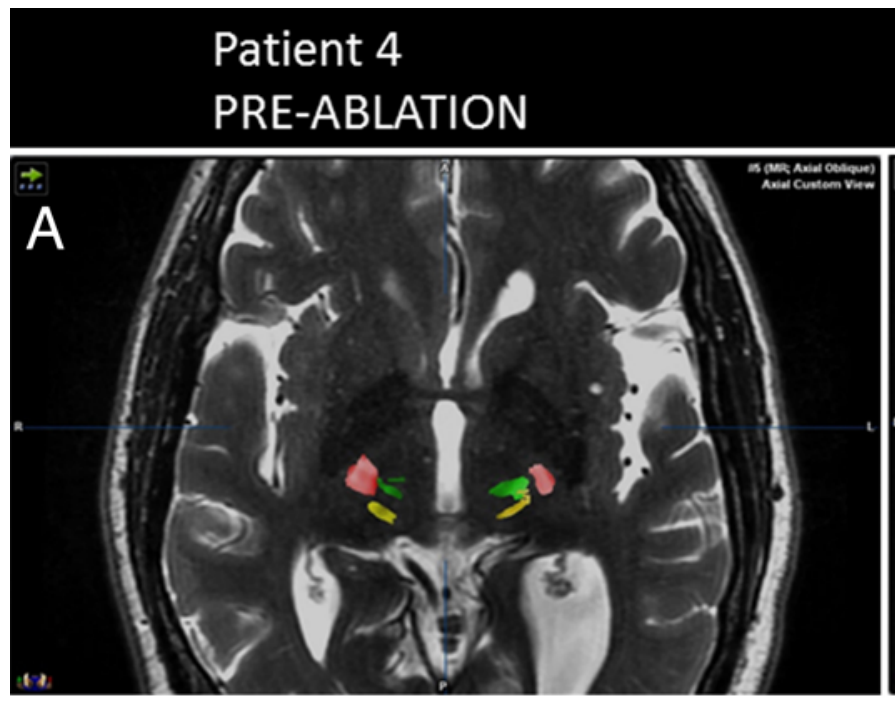

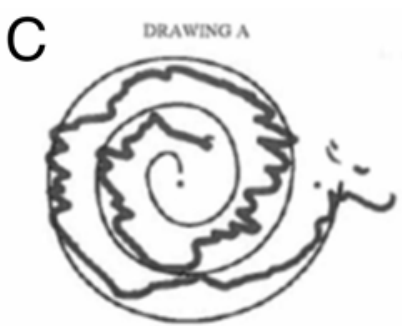

Dawnoc

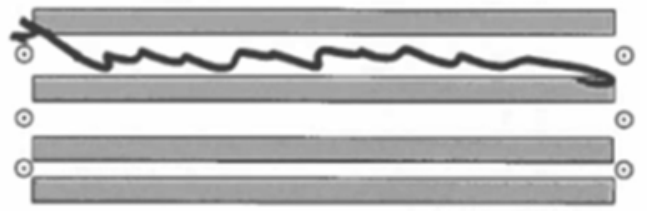

POST-ABLATION

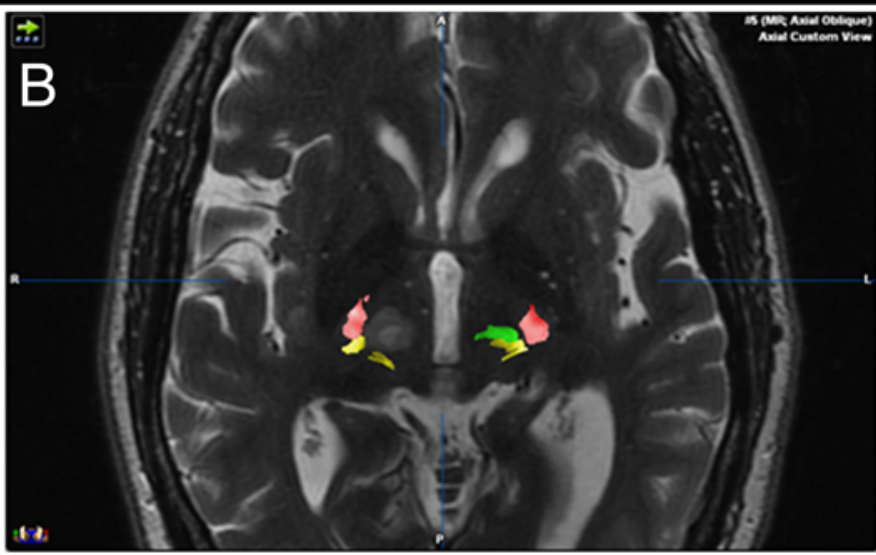

$\mathrm{D}$
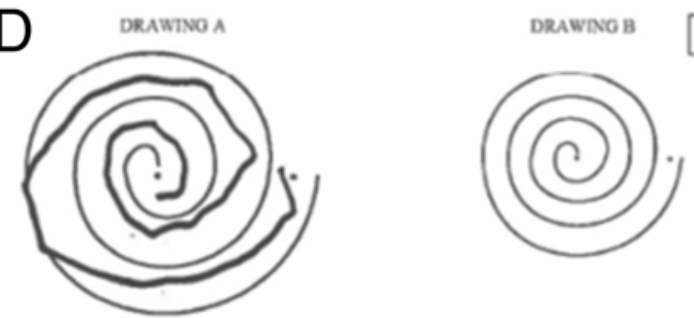

DRAwNoc

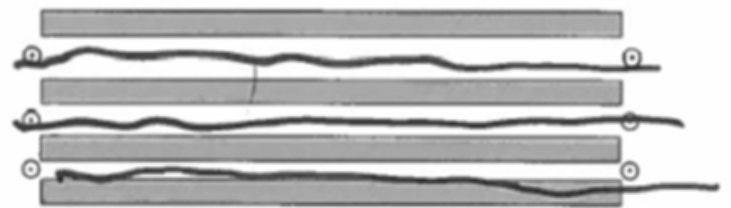

FIG. 6. A and B: Pre- and postablation DTI tractography overlaid on T2-weighted sequences for Case 4. CST (red), ML (yellow), and corticothalamic tract (green) are shown from DTI. Postablation imaging reveals the T2-weighted hyperintense MRgFUS lesion in the location of the right DRT that could no longer be successfully tracked. C and D: Results of the pre- and postprocedure Archimedes spiral drawing tests.

information helpful when making intraprocedural adjustments.

Both the immediate postablation and postoperative Day 1 MRI examinations revealed interruption of the DRT ipsilateral to the ablation side by DTI evaluation. The authors believe the immediate postprocedure MRI is scientifically the most relevant because edema has not peaked and no susceptibility change is identified at this point to indicate hemorrhage that might otherwise disrupt the fiber tracking. In contrast, the postoperative Day 1 MRI showed increased edema and petechial hemorrhage (Fig. 1), consistent with findings from other authors. ${ }^{19}$ The postoperative Day 1 DTI was also processed and revealed identical tract disruption in all patients.

Limitations of this study include the small sample size and limited number of patients. In particular, true statistical correlation between outcome and an imaging biomarker such as DRT DTI would require a range of clinical and radiographic responses, and the similar clinical and radiographic findings in this small series precludes such an analysis. Future studies on larger numbers of patients should help clarify this, as will application of this technique to patients with clinical failures or tremor recurrence from any ablative or neuromodulation procedure, prior to considering MRgFUS thalamotomy. FDA clearance of certain DBS systems for use in the MRI may also facilitate similar studies in patients with existing DBS systems who have good ongoing benefit as well as patients with failure, to determine the ultimate utility of this method to troubleshoot difficult cases.

\section{Conclusions}

In this study we present a limited cohort of patients with ET who underwent successful MRgFUS of the Vim thalamus with excellent clinical response. Pre- and postprocedure DTI aided in optimal selection of ablation site and showed DRT disruption immediately following the 
procedure. We believe DTI-based functional imaging techniques provide significant benefits in procedural planning, performance, and follow-up.

\section{Acknowledgments}

We would like to acknowledge Dr. Gary Dorfman, Kristin Strybing, Alyson Hignight, Muc Du, and Jojo Borja for their contributions to this work.

\section{References}

1. Benabid AL, Pollak P, Gervason C, Hoffmann D, Gao DM, Hommel M, et al: Long-term suppression of tremor by chronic stimulation of the ventral intermediate thalamic nucleus. Lancet 337:403-406, 1991

2. Chang WS, Jung HH, Kweon EJ, Zadicario E, Rachmilevitch I, Chang JW: Unilateral magnetic resonance guided focused ultrasound thalamotomy for essential tremor: practices and clinicoradiological outcomes. J Neurol Neurosurg Psychiatry 86:257-264, 2015

3. Chang WS, Jung HH, Zadicario E, Rachmilevitch I, Tlusty $\mathrm{T}$, Vitek S, et al: Factors associated with successful magnetic resonance-guided focused ultrasound treatment: efficiency of acoustic energy delivery through the skull. J Neurosurg 124:411-416, 2016

4. Coenen VA, Allert N, Mädler B: A role of diffusion tensor imaging fiber tracking in deep brain stimulation surgery: DBS of the dentato-rubro-thalamic tract (drt) for the treatment of therapy-refractory tremor. Acta Neurochir (Wien) 153:1579-1585, 2011

5. Deuschl G, Raethjen J, Hellriegel H, Elble R: Treatment of patients with essential tremor. Lancet Neurol 10:148-161, 2011

6. Elble R, Bain P, Forjaz MJ, Haubenberger D, Testa C, Goetz $\mathrm{CG}$, et al: Task force report: scales for screening and evaluating tremor: critique and recommendations. Mov Disord 28:1793-1800, 2013

7. Elias WJ, Huss D, Voss T, Loomba J, Khaled M, Zadicario E, et al: A pilot study of focused ultrasound thalamotomy for essential tremor. N Engl J Med 369:640-648, 2013

8. Elias WJ, Lipsman N, Ondo WG, Ghanouni P, Kim YG, Lee $\mathrm{W}$, et al: A randomized trial of focused ultrasound thalamotomy for essential tremor. N Engl J Med 375:730-739, 2016

9. Jenne JW: Non-invasive transcranial brain ablation with high-intensity focused ultrasound. Front Neurol Neurosci 36:94-105, 2014

10. Khanna N, Gandhi D, Steven A, Frenkel V, Melhem E: Intracranial applications of MR imaging-guided focused ultrasound. AJNR Am J Neuroradiol 38:426-431, 2017

11. Kim MJ, Jeon SR, Kim SR, Lee MC, Chung SJ: Lateralized effects of unilateral thalamotomy and thalamic stimulation in patients with essential tremor. J Mov Disord 4:64-67, 2011

12. Lipsman N, Schwartz ML, Huang Y, Lee L, Sankar T, Chap- man M, et al: MR-guided focused ultrasound thalamotomy for essential tremor: a proof-of-concept study. Lancet Neurol 12:462-468, 2013

13. Louis ED: Utility of the hand-drawn spiral as a tool in clinical-epidemiological research on essential tremor: data from four essential tremor cohorts. Neuroepidemiology 44:45-50, 2015

14. Lozano AM: Vim thalamic stimulation for tremor. Arch Med Res 31:266-269, 2000

15. Rieke V, Butts Pauly K: MR thermometry. J Magn Reson Imaging 27:376-390, 2008

16. Rieke V, Instrella R, Rosenberg J, Grissom W, Werner B, Martin E, et al: Comparison of temperature processing methods for monitoring focused ultrasound ablation in the brain. $\mathbf{J}$ Magn Reson Imaging 38:1462-1471, 2013

17. Sammartino F, Krishna V, King NK, Lozano AM, Schwartz ML, Huang Y, et al: Tractography-based ventral intermediate nucleus targeting: novel methodology and intraoperative validation. Mov Disord 31:1217-1225, 2016

18. Vayssiere N, Hemm S, Cif L, Picot MC, Diakonova N, El Fertit $\mathrm{H}$, et al: Comparison of atlas- and magnetic resonance imaging-based stereotactic targeting of the globus pallidus internus in the performance of deep brain stimulation for treatment of dystonia. J Neurosurg 96:673-679, 2002

19. Wintermark M, Druzgal J, Huss DS, Khaled MA, Monteith $\mathrm{S}$, Raghavan P, et al: Imaging findings in MR imaging-guided focused ultrasound treatment for patients with essential tremor. AJNR Am J Neuroradiol 35:891-896, 2014

20. Yamada K, Akazawa K, Yuen S, Goto M, Matsushima S, Takahata A, et al: MR imaging of ventral thalamic nuclei. AJNR Am J Neuroradiol 31:732-735, 2010

\section{Disclosures}

Financial support for this study was provided by Insightec (Haifa, Israel).

\section{Author Contributions}

Conception and design: Kaplitt, Chazen, Stieg, Min. Acquisition of data: Kaplitt, Chazen, Sarva, Pryor, Riegelhaupt. Analysis and interpretation of data: Kaplitt, Chazen, Sarva. Drafting the article: Kaplitt, Chazen, Sarva. Critically revising the article: all authors. Reviewed submitted version of manuscript: all authors. Approved the final version of the manuscript on behalf of all authors: Kaplitt. Administrative/technical/material support: Kaplitt, Chazen, Stieg, Min, Ballon. Study supervision: Kaplitt, Chazen.

\section{Correspondence}

Michael G. Kaplitt, Department of Neurosurgery, Weill Cornell Medicine, NewYork-Presbyterian Hospital, 525 East 68th St., Box 99, New York, NY 10065. email: mik2002@med.cornell.edu. 